\title{
The efficacy of a Mediterranean type diet on symptoms of osteoarthritis - a pilot study
}

\author{
G. Davison, J. R. Dyer, S. M. Marcora and A. R. Mauger \\ School of Sport and Exercise Sciences, Faculty of Science, University of Kent, ME4 4AG, UK
}

Osteoarthritis (OA) is a group of chronic, painful, disabling conditions affecting synovial joints where the pathology includes damage and loss of articular cartilage, irregular remodelling and erosion of sub-articular bone, synovial distension and inflammation ${ }^{(1)}$. Whilst current management strategies include exercise, weight loss and pharmacological therapies, there is evidence to suggest that particular food sources consistent with a Mediterranean type diet (e.g. rich in omega-3 fatty acid) may slow the pathogenesis of degenerative joint diseases ${ }^{(2)}$ and moderate markers of inflammation ${ }^{(3)}$. Consequently, following a diet which is plant based, favours olive oil over animal fats, and is high in fibre, vegetables, and fruits, combined with a low intake of foods which are associated with increased inflammation (e.g. meat, dairy, fat and sugar) may reduce joint degradation and inflammation associated with OA. The primary aim of the present pilot study was to assess whether following a Mediterranean type diet would moderate the physiological and perceptual symptoms of participants diagnosed with OA.

Following full institutional ethical approval according to the guidelines laid down in the Declaration of Helsinki, 45 participants diagnosed with OA were randomly allocated to an experimental or control group to participate in either Part A or Part B of this 4 month study. In Part A (EXP = 19; CON =6), participants completed the previously validated Arthritis Impact Measurement Scales Health Status Questionnaire (AIMS2) and a 1 week food frequency questionnaire on enrolment, halfway through the study (month 2) and at the end of the study (month 4). In Part B $(\mathrm{EXP}=13 ; \mathrm{CON}=7)$, participants provided the same data but gave a venous blood sample on enrolment and at month 4 for analysis of serum cartilage oligomeric matrix protein (sCOMP) (a marker of cartilage degradation) instead of the AIMS2 questionnaires. The CON groups were asked to maintain their usual activities and dietary practices, whereas the EXP groups were asked to follow a dietary practice which was consistent with a Mediterranean type diet.

Participants in the EXP group demonstrated lower levels of sCOMP from pre- to post-intervention (pre- $=14.1 \pm 4.9 \mathrm{U} / \mathrm{L}$; post- $=$ $13 \cdot 1 \pm 5 \cdot 2 \mathrm{U} / \mathrm{L}$, Cohen's $d=0.413)$, although this was not significant $(p>0.05)$. Participants in the CON group experienced no change in sCOMP from pre- to post-intervention (pre- $=9 \cdot 6 \pm 1.4 \mathrm{U} / \mathrm{L}$ post- $=10 \cdot 0 \pm 2.4 \mathrm{U} / \mathrm{L}$, Cohen's $d=-0 \cdot 154$ ). None of the components of the AIMS2 questionnaire were changed over the 4-month study in the CON group, but the Affect component significantly decreased by $19 \%$ from pre- to post dietary intervention $(p=0.02)$ in the EXP group.

The results of this pilot study indicate that a 4-month dietary intervention which prescribes a Mediterranean type diet for patients diagnosed with OA results in a significant improvement in the perceptual markers of OA and a moderate reduction in sCOMP - a marker of cartilage degradation. These findings suggest that simple modifications to diet may help patients with OA manage the progression of their disease and improve their quality of life. The findings of this study should be supported with an increased sample size to statistically confirm the observed meaningful effect sizes, and a greater range of biomarker analysis (both inflammatory and degradation markers) to assess the impact on the physiological progression of OA.

1. Hutton CW (1989) Ann Rheum Dis 48, 958-961.

2. Knott, Avery NC, Hollander AP, et al. (2011) Osteoarthritis Cartilage 19, 1150-1157.

3. Dean E \& Hansen RG (2012) Arthritis 2012, 1-28. 